\title{
Heisenberg Model of a Ferromagnetic Monolayer Deposited on a Non-Magnetic Bulk Substrate
}

\author{
W. JAWORSKI \\ Quantum Physics Division, Faculty of Physics, Adam Mickiewicz University \\ Umultowska 85, 61-614 Poznań, Poland
}

\begin{abstract}
A model describing a Heisenberg ferromagnetic monolayer (in a magnetic field) interacting with its nonmagnetic bulk substrate is formulated. We use reduced-density operators to show that physical properties of the monolayer are affected by its interaction with the environment. Particularly the influence of the substrate lattice vibrations on the monolayer exchange parameter is examined. The Gaussian-type orbitals were used to calculate the distance dependence of the exchange parameter and the many-body Green functions to calculate the temperature dependence of the magnetization. Finally, the influence of the Debye temperature of the substrate on the magnetization of the monolayer is depicted. Although the resultant effect is not prominent, we state that interaction of ultrathin magnetic films with their environment has to be taken into account in the construction of the reduced-density operator.
\end{abstract}

PACS numbers: 75.10.Dg, 75.10.Hk, 75.10.Jm

\section{Introduction}

Ultrathin magnetic films are objects of great interest, including the theoretical point of view. Several models have been proposed and studied using many theoretical methods to predict and explain the properties of the films. However, in experimental and technological applications the films are very often deposited on bulk substrates with which they surely interact. What is more, the number of interacting particles is frequently of the same order of magnitude as the total number of particles in the film. Therefore we claim that this interaction cannot be neglected and we may use the reduced-density operators, which include the interaction [1]. These operators are derived on the basis of an approach called quantum thermodynamics [2], which has proven to be successful in showing that the interaction with environment can significantly change the physical properties of ultrathin films [3].

\section{Model}

\subsection{Reduced-density operators}

According to [1], we may write the reduced-density operator of an ultrathin film, denoted by $j$, interacting with its bulk substrate, $j^{\prime}$, in the form

$$
d_{j}=\exp \left(\beta\left(F_{j}-H_{j}-H_{j}^{\prime}\right)\right),
$$

where $F_{j}$ and $H_{j}$ stand for the films' free energy and Hamiltonian, respectively, while $\beta=\frac{1}{k_{\mathrm{B}} T}$ with $k_{\mathrm{B}}$ being the Boltzmann constant and $T$ - temperature. The $H_{j}^{\prime}$ expression describes the effect of the interaction taken into regard in the mean field-type approximation [1]:

$$
H_{j}^{\prime} \simeq\left\langle H_{j j^{\prime}}\right\rangle_{j^{\prime}}=\operatorname{Tr}_{j^{\prime}}\left[H_{j j^{\prime}} d_{j^{\prime}}\right] \text {, }
$$

where $H_{j j^{\prime}}$ is the Hamiltonian describing the interaction between systems $j$ and $j^{\prime}$. The mean value $\langle\ldots\rangle_{j^{\prime}}$ and the partial trace $\operatorname{Tr}_{j^{\prime}}[\ldots]$ are calculated in the states of the substrate's Hamiltonian $H_{j^{\prime}}$, with the reduced-density operator $d_{j^{\prime}}$ having the form similar to (1), but with no terms describing the interaction, as it is only a small surface effect.

\subsection{Hamiltonians}

For more detailed calculations, we consider the thin film to be a monolayer of spins $S=\frac{1}{2}$ described by the ferromagnetic Heisenberg Hamiltonian

$$
H_{j}=-B \sum_{f} S_{f}^{z}-\frac{1}{2} \sum_{f \neq f^{\prime}} J \boldsymbol{S}_{f} \cdot \boldsymbol{S}_{f^{\prime}}
$$

where $B$ is the magnetic field in the $z$-direction (perpendicular to the film $x y$-plane), the summation runs over neighbouring lattice sites and $J>0$ is the exchange parameter. The spin operators $\boldsymbol{S}_{f}=\left(S_{f}^{x}, S_{f}^{y}, S_{f}^{z}\right)$ for $S=\frac{1}{2}$ obey the usual commutation rules (with $\hbar=1$ )

$$
\left[S_{f}^{k}, S_{f^{\prime}}^{l}\right]=\mathrm{i} \delta_{f f^{\prime}} \varepsilon_{k l m} S_{f^{\prime}}^{m}
$$

We also assume that the monolayer is epitaxially deposited on a bulk non-magnetic simple cubic crystal, having $N_{a}$ atoms of mass $m$. Its Hamiltonian $H_{j^{\prime}}$ is written as

$$
H_{j^{\prime}}=\sum_{\boldsymbol{q}, p} \omega_{\boldsymbol{q}, p}\left(n_{\boldsymbol{q}, p}+\frac{1}{2}\right),
$$

where $n_{\boldsymbol{q}, p}=a_{\boldsymbol{q}, p}^{\dagger} a_{\boldsymbol{q}, p}$ is the phonon number operator, 
while the operators $a_{\boldsymbol{q}, p}^{\dagger}$ and $a_{\boldsymbol{q}, p}$ create and annihilate a phonon (with a wave vector $\boldsymbol{q}$ and polarisation $p$ ), respectively, and they obey the bosonic commutation rules

$$
\left[a_{\boldsymbol{q}, p}, a_{\boldsymbol{q}^{\prime}, p^{\prime}}^{\dagger}\right]=\delta_{\boldsymbol{q} \boldsymbol{q}^{\prime}} \delta_{p p^{\prime}}
$$

Finally, the Hamiltonian describing the interaction between systems $j$ and $j^{\prime}$ is taken in the form

$$
H_{j j^{\prime}}=-\frac{1}{2} \sum_{f \neq f^{\prime}}\left(J_{1} \boldsymbol{u}_{g}+J_{2} \boldsymbol{u}_{g}^{2}\right) \boldsymbol{S}_{f} \cdot \boldsymbol{S}_{f^{\prime}},
$$

where $\boldsymbol{u}_{g}$ is a displacement field operator, given in a form similar to that in [4]:

$$
\begin{gathered}
\boldsymbol{u}_{g}=\sum_{\boldsymbol{q}, p, \alpha} \boldsymbol{e}_{\boldsymbol{q}, p}^{\alpha} \sqrt{\frac{1}{2 N_{a} m \omega_{\boldsymbol{q}, p}}} \\
\times\left(a_{\boldsymbol{q}, p} \mathrm{e}^{\mathrm{i} \boldsymbol{q} \cdot \boldsymbol{r}_{g}}+a_{\boldsymbol{q}, p}^{\dagger} \mathrm{e}^{-\mathrm{i} \boldsymbol{q} \cdot \boldsymbol{r}_{g}}\right),
\end{gathered}
$$

where $\boldsymbol{e}_{\boldsymbol{k}, p}^{\alpha}(\alpha=x, y, z)$ are the unit vectors.

\section{Calculations}

\subsection{Mean quadratic displacement}

If we calculate the Hamiltonian $H_{j}^{\prime}$, using the eigenstates $\left|n_{\boldsymbol{q}, p}\right\rangle$ of the phonon number operator $n_{\boldsymbol{q}, p}$, we will see that the mean value of the displacement operator given by (8) vanishes: $\left\langle\boldsymbol{u}_{g}\right\rangle_{j^{\prime}}=0$. To obtain the mean quadratic displacement we use the Debye approximation as in [4] and we get

$$
\left\langle\boldsymbol{u}_{g}^{2}\right\rangle_{j^{\prime}}=\frac{9}{m k_{\mathrm{B}} \Theta}\left[\frac{1}{4}+\frac{T}{\Theta} \Phi\left(\frac{\Theta}{T}\right)\right],
$$

where $\Theta$ is the Debye temperature of the substrate and $\Phi(x)$ is the Debye function given by

$$
\Phi(x)=\frac{1}{x} \int_{0}^{x} \mathrm{~d} y \frac{y}{\mathrm{e}^{y}-1} .
$$

It may be now noticed that the Hamiltonian given by

$$
H=H_{j}+H_{j}^{\prime}=-B \sum_{f} S_{f}^{z}-\frac{1}{2} \sum_{f \neq f^{\prime}} J_{\mathrm{eff}} \boldsymbol{S}_{f} \cdot \boldsymbol{S}_{f^{\prime}}
$$

includes the effect of thermal vibrations of the substrate's lattice on the monolayer via the effective exchange parameter

$$
J_{\text {eff }}=J+J_{2}\left\langle\boldsymbol{u}_{g}^{2}\right\rangle_{j^{\prime}} .
$$

\subsection{Exchange parameter distance dependence}

In order to estimate the values $J$ and $J_{2}$ in (12), we numerically calculate the exchange parameter as a function of the lattice constant. To do that we assume that every spin $S_{f}=\frac{1}{2}$ is described by a one-electron wave function in the form of a Gaussian-type orbital centered at $\boldsymbol{R}_{f}=\left(x_{f}, y_{f}, z_{f}\right)$ :

$$
\phi_{f}(\boldsymbol{r})=\left(z-z_{f}\right)^{2} \exp \left(-\alpha\left(\boldsymbol{r}-\boldsymbol{R}_{f}\right)^{2}\right) .
$$

For simplicity, we perform the calculations for only $N_{s}=4$ sites forming a square and choose $\alpha=0.1$. We use the Löwdin-Carr method presented in $[5,6]$ to obtain energies in two states described by the Slater determinantal wave functions: the ferromagnetic state energy

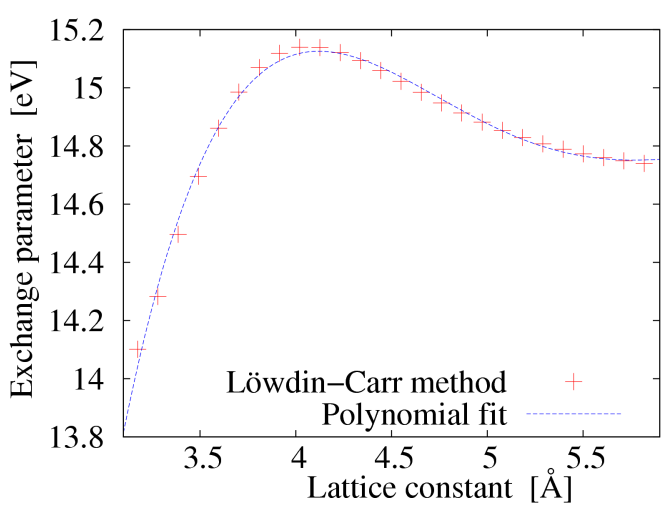

Fig. 1. The exchange parameter as a function of the lattice constant calculated using the Löwdin-Carr method and the obtained polynomial fitting curve.

$E_{\mathrm{F}}$ and the antiferromagnetic (Néel) state energy $E_{\mathrm{AF}}$. The corresponding one- and two-electron integrals are calculated according to the properties of Gaussian-type orbitals presented in $[7,8]$. Finally, we evaluate the exchange parameter $J_{\mathrm{LC}}$ for a ceratain lattice constant $a_{0}$ from

$$
E_{\mathrm{AF}}-E_{\mathrm{F}}=\frac{N_{n}}{2} J_{\mathrm{LC}},
$$

where $N_{n}=4$ is the number of nearest neighbours. The results for various lattice constants are presented in Fig. 1. We are now able to expand the exchange parameter $J_{\mathrm{LC}}(a)$ about a certain lattice constant $a_{0}$ and get

$$
J=J_{\mathrm{LC}}\left(a_{0}\right), \quad J_{2}=\frac{1}{2}\left(\frac{\partial^{2}}{\partial a^{2}} J_{\mathrm{LC}}\right)\left(a_{0}\right) .
$$

\subsection{Magnetization}

To obtain the magnetization $\left\langle S^{z}\right\rangle$ of the $\operatorname{spin} S=\frac{1}{2}$ monolayer in the mean field theory (MFT) approximation, we use the many-body Green functions presented in [9]. Following a couple of calculations, we get the equation for the magnetization in the form

$$
\left\langle S^{z}\right\rangle=\frac{1}{2} \tanh \frac{B+4\left\langle S^{z}\right\rangle J_{\mathrm{eff}}}{2 k_{\mathrm{B}} T},
$$

which may be solved self-consistently.

Results obtained for two different lattice constants which coincide with different values of the parameters given in (15) are presented in Fig. 2.

TABLE

The Curie temperatures $T_{\mathrm{C}}$ of the ferromagnetic monolayer obtained from (16) for different substrate's Debye temperatures $\Theta$ and lattice constants $a_{0}$.

\begin{tabular}{c|c|c|c|c|c|c}
\hline \hline$a_{0}[\AA]$ & \multicolumn{3}{|c|}{3.5} & \multicolumn{3}{c}{4.0} \\
\hline$\Theta[\mathrm{K}]$ & 50 & 250 & 450 & 50 & 250 & 450 \\
$T_{\mathrm{C}}[\mathrm{eV}]$ & 14.54 & 14.71 & 14.72 & 15.01 & 15.10 & 15.11
\end{tabular}




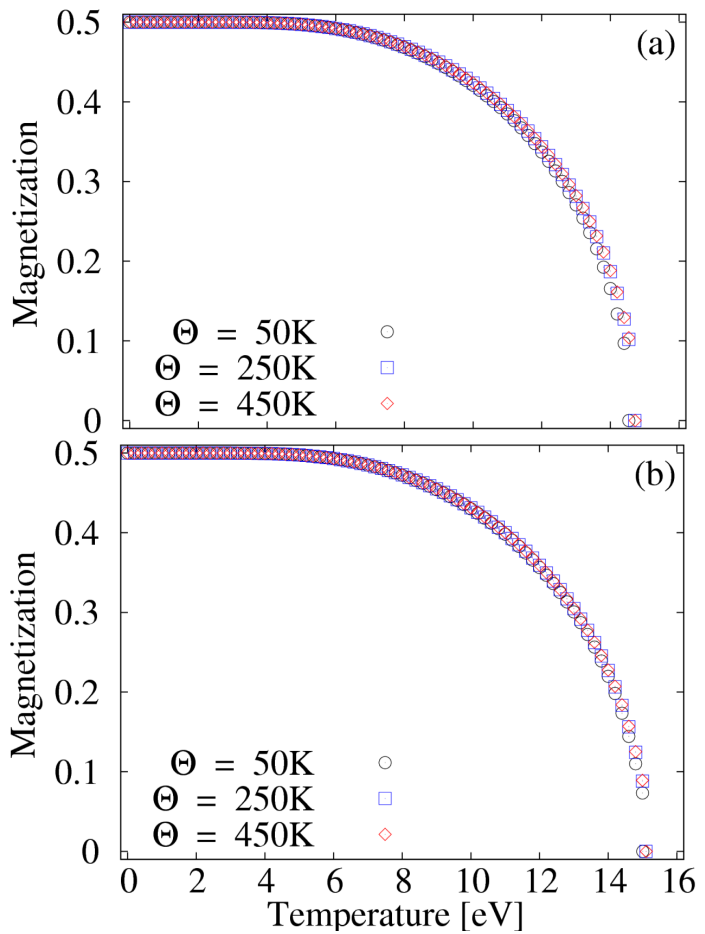

Fig. 2. The temperature dependences of the magnetization $\left\langle S^{z}\right\rangle$ without the magnetic field $(B=0)$ plotted for different substrate's Debye temperatures $\Theta$ and lattice constants: (a) $a_{0}=3.5 \AA$ and (b) $a_{0}=4.0 \AA$.

What is more, using the fact that the MFT approximation violates the Mermin-Wagner theorem [9, 10], we can calculate the Curie temperature of the monolayer. We expand the left hand side of (16) for small $\left\langle S^{z}\right\rangle$ and self-consistently evaluate the Curie temperature from $T_{\mathrm{C}}=J_{\text {eff }}\left(T_{\mathrm{C}}\right)$ for different substrate's Debye temperatures $\Theta$ and lattice constants $a_{0}$. The results are shown in Table.

\section{Conclusions}

The results depicted in Fig. 2 and in Table show that the behaviour of the monolayer's magnetization does not alter much with the change of the substrate's Debye temperature. This is due to the mean quadratic displacement $\left\langle\boldsymbol{u}_{g}^{2}\right\rangle_{j^{\prime}} \approx 10^{-2} \AA$ and the value of the $J_{2}$ parameter from (15). Considering an exchange parameter $J_{\text {eff }}$ with a stronger distance dependence could have had a more prominent effect. Moreover, taking into regard various types of the substrate and the interaction Hamiltonians (5), (7) could be helpful in finding how the substrate influences the film and verifying it experimentally.

We hope that more refined calculations with the use of reduced-density operators may become useful in the investigation of the interaction and correlation between ultrathin magnetic films and their substrates.

\section{References}

[1] Z. Jacyna-Onyszkiewicz, Physica A 305, 497 (2002).

[2] Z. Jacyna-Onyszkiewicz, The Principles of Quantum Thermodynamics, UAM, Poznań 1986 (in Polish).

[3] Z. Jacyna-Onyszkiewicz, A. Wierzbicki, Phys. Status Solidi B 188, K 17 (1995).

[4] R.J. Glauber, Phys. Rev. 98, 1692 (1995).

[5] W.J. Carr, Phys. Rev. 92, 1 (1953).

[6] D.C. Mattis, The Theory of Magnetism I, Springer, Berlin 1981, p. 60.

[7] J. Kohanoff, Electronic Structure Calculations for Molecules and Solids, Cambridge University Press, Cambridge 2006, p. 196.

[8] T. Helgaker, P. Jørgensen, J. Olsen, Molecular Electronic-Structure Theory, Wiley, Chichester 2000, p. 287.

[9] P. Fröbrich, P.J. Kuntz, Phys. Rep. 432, 223 (2006).

[10] N.D. Mermin, H. Wagner, Phys. Rev. Lett. 17, 22 (1966). 